\title{
FACTORES ASOCIADOS AL RENDIMIENTO ACADÉMICO EN ESTUDIANTES DE PSICOLOGÍA DE LA UNMSM
}

\author{
Lupe García Ampudia \\ Oswaldo Orellana Manrique \\ Isaac Canales Quevedo
}

En el presente trabajo se estudia los factores relacionados con el rendimiento académico en un grupo de estudiantes universitarios de la facultad de psicología de la UNMSM, entre los factores se ha considerado la motivación, las estrategias de aprendizaje y la autoestima y en lo correspondiente al rendimiento académico se ha considerado el promedio de notas obtenido por los alumnos en los tres primeros ciclos de estudio.

La muestra estudiada estuvo constituida por los ingresantes en el año 1999 a la facultad de psicología, los instrumentos utilizados fueron: el cuestionario de estrategias de aprendizaje y motivación adaptado al español por C. Roces, J. Touron y M. Gonzáles, y el inventario de auto estima de Rosenberg, para el rendimiento académico siguiendo Roces, Touron y Gonzáles (1995) se consideró tres niveles de rendimiento: alto, medio y bajo, considerando los percentiles como punto de corte.

Los resultados indican la existencia de correlaciones inversas entre la orientación a metas extrínsecas y ansiedad con el rendimiento académico y una correlación positiva entre la estrategia, la constancia y el rendimiento, no se encontró relación entre la autoestima y el rendimiento.

En la comparación de grupos de distinto nivel de rendimiento se encontraron diferencias significativas en la escala de orientación a metas extrínsecas en los grupos de alto y bajo rendimiento; también se encontraron diferencias en la escala de ansiedad, creencias de control y autoeficacia para el aprendizaje, presentándose una relación inversa entre éstas escalas y el rendimiento, es decir a mayor rendimiento, menor ansiedad, creencias de control y auto eficacia para el aprendizaje, en cambio se encontró una relación positiva entre la constancia y el rendimiento.

En cuanto a estrategias de aprendizaje se encontraron diferencias significativas en la escala de aprovechamiento de tiempo, donde los alumnos con alto rendimiento valoran menos el aprovechamiento de tiempo y la concentración que los alumnos de bajo rendimiento, si bien las diferencias no son significativas se encontró relaciones entre rendimiento y las escalas de organización, constancia y meta cognición.

Con relación a la autoestima y el rendimiento, no se encontraron diferencias significativas, sin embargo los alumnos de rendimiento alto obtuvieron niveles medios más altos de autoestima que los de bajo rendimiento.

PALABRAS CLAVE: rendimiento académico. estrategias de aprendizaje. motivación. autoestima. 
In the present job is studied the factors related to the academic performance in to university group of students of the Faculty of Psychology of the UN MSM, among the factors has been considered the motivation, the strategies of learning and the self-esteem and in it pertaining to the academic performance the average of notices there is been considered obtained by the students in the three first cycles of study. The sample studied was constituted by the ingresantes in the year 1,999 to the Faculty of Psychology, the instruments utilized were: the Learning Strategies Questionnaire and Motivation adapted the Spaniard by C. GRAZE, J. TOURON AND M. Gonzales, and the inventory of Selfesteem of Rosenberg, for the academic performance following Graze, Touron and Gonzales (1995) was considered three levels of performance: high, medium and under, considering the percentiles ace point of cut. The results indicates you the in verse existence of correlation among the orientation to extrinsic goals and anxiety with the academic performance and to positive correlation among the strategy: constancy and the performance, itself not relation was found among the self-esteem and the performance. In the comparison of groups of distinct level of performance they were found differentiates significant in the scale of orientation to extrinsic goals in the groups of high and under performance; al so differences in the scale of anxiety were found, beliefs of control and autoeficacia for the learning, being presented and inverse relation among these scales and the performance, that is to say to greater performance, smaller anxiety, beliefs of control and autoeficacia for the learning, on the other hand to positive relation among the constancy was found and the performance. Ace for strategies of learning they were found differentiates significant in the scale of use of cheats, where the students with high performance valued except the use of it cheats and the concentration that the students of under performance, though the differences it plows not significant was found relations among performance and the scales of organization, constancy and metacognición. With relation to the self-esteem and the performance, themselves they were not found differentiates significant, however the high students of performance obtained higher medium levels of self-esteem that the of under performance.

KEY WORDS: Academic Performance, Strategies of Learning, Motivation, Self-esteem, 


\section{INTRODUCCIÓN}

Uno de los problemas que atraviesa la educación en sus diferentes niveles es el del rendimiento académico; sin embargo la mayor atención se ha centrado en el nivel básico, y a diferencia de otros países el nivel superior en nuestro País no ha merecido la atención ni de los organismos responsables ni de los investigadores.

El aumento de la demanda de la educación superior a partir de la década del 60, masificó la educación y trajo consigo problemas de rendimiento académico que se expresaron en fracaso escolar, deserción, prolongación de estudios etc; pese que aún no se han superado éstos problemas, las instituciones educativas el día de hoy se plantean como uno de sus objetivos estratégicos mejorar la calidad de la educación, lo cual inevitablemente nos conduce al tema del rendimiento académico que sin ser el único indicador de calidad es uno de los más importantes (Marchesi y Mal1in 1999).

Desde el punto de vista teórico cuando se ha estudiado los factores vinculados al aprendizaje y el rendimiento académico, tradicional-mente ha existido una separación entre los factores cognitivos y afectivo-motivacionales, expresión de uno de los dualismos que afectó y aún afecta aunque en menor medida a la psicología (Marchesi y Mallín, 1999) unos investigadores centraron sus estudios enfatizando el papel decisivo de los factores cognitivos, subestimando los factores afectivo motivacionales; otros en cambio, enfatizaban el papel de los factores afectivo motivacionales relegando a un segundo plano los factores cognitivos.

Después de la década del 80 y a la luz de los trabajos realizados en la psicología cognitiva se intensifica el interés por el estudio de la influencia de las variables motivacionales y cognitivas en el aprendizaje y rendimiento académico encontrándose una estrecha relación entre ellas (Weiner,1988, Pintrich y Schrauben, 1992, Mckeachie y cols.,1986) Así también las nuevas concepciones de aprendizaje enfatizan una visión más holistica e integradora y en la que igualmente se consideran no sólo aspectos cognitivos y metacognitivos, sino también aspectos afectivo-motivacionales, y es el modelo de aprendizaje autorregulado de Mckeachie, Pintrich y sus colaboradores (1992) quienes demuestran que los factores cognitivos, motivacionales y sus relaciones los que ejercen una influencia directa en la implicación del estudiante en el aprendizaje y rendimiento académico.

En suma, se llega a la conclusión que para aprender no basta con poseer las capacidades necesarias, también hace falta querer hacerlo, lo que implica tener la disposición y motivación correspondiente; pero igualmente para aprender no basta estar motivado, porque ésta se ve afectada cuando el esfuerzo desplegado para aprender (por falta de estrategias) resulta insuficiente y no conduce a los resultados esperados.

Las estrategias de aprendizaje

consideradas como una variable cognitiva son un conjunto de procedimientos que un alumno adquiere y emplea de forma intencional y está dirigida a un objetivo determinado, en tal sentido se consideran como una guía para las acciones (Monereo.1997).

Según Monereo (1997) son procesos de toma de decisiones concientes e intencionales, en los cuales el alumno elige y recupera, de manera coordinada, los conocimientos que necesita para cumplir una determinada demanda u objetivo, dependiendo de las características de la situación educativa en que se produce la acción. 
Un alumno emplea estrategias de aprendizaje cuando es capaz de ajustar su compollamiento, a las exigencias de una tarea y a las circunstancias en que se produce esa demanda, lo que a su vez implica que los objetivos educativos que el profesor se propone no se queden en la mera reproducción o réplica de datos.

Aprender estratégicamente no depende del conocimiento de un conjunto de técnicas de estudio, sino de la posibilidad de captar las exigencias de las tareas en una situación de aprendizaje determinada y controlar con los medios adecuados dicha situación. "el factor que distingue un buen aprendizaje de otro malo o inadecuado es la capacidad de examinar las situaciones. las tareas y los problemas y responder en consecuencia; ésta capacidad raras veces es enseñada o alentada en la escuela" ( Nisbet y Shucksmith, 1986, pago 47).

La optimización del rendimiento académico requiere del conocimiento y empleo de estrategias por parte del aprendiz, es decir no se trata solamente de conocer un conjunto de estrategias sino de actuar estratégicamente, lo que implica saber cuando, donde y en qué utilizarlas, en otras palabras saber actuar estratégicamente, es entonces obligación de las instituciones educativas no sólo enseñar estrategias sino tener profesores estratégicos, que planifiquen las actividades de aprendizaje, única garantía para que los alumnos "aprendan a aprender".

La autoestima entendida como la valoración personal del sujeto sobre la base de sus experiencias y su interacción con los "otros significativos" se convierte en un mecanismo regulador de la conducta por cuanto el sujeto anticipa sus resultados a partir de las valoraciones que hace de sus capacidades lo que a su vez genera expectativas de éxito o de fracaso, en el caso del estudiante una desvalorización subjetiva de sus capacidades puede orientarlo a un bajo rendimiento académico por cuanto el mismo no tiene confianza en sus posibilidades. A su vez un rendimiento por debajo de lo esperado puede afectar la autoestima del alumno, las investigaciones llevadas a cabo acerca de las relaciones entre la autoestima y el rendimiento han arrojado resultados dispares y contradictorios. pero como señala Nuñez y González Pumariega (1996) es posible que los mismos se deban a la presencia de otras variables en el rendimiento académico.

En la facultad de psicología de la UNMSM nos interesa elevar el nivel de rendimiento académico de los alumnos, por ello nos parece importante investigar el tema en todas sus dimensiones, y para el presente trabajo nos planteamos las siguientes interrogantes:

¿Cuáles son los aspectos motivacionales y de estrategias donde los alumnos de psicología obtienen puntuaciones más altas y bajas?

¿Cuáles son las diferencias entre motivación, estrategias de aprendizaje y autoestima en estudiantes de psicología de diferente nivel de rendimiento?

¿Cuál es la relación entre la motivación. las estrategias de aprendizaje y la autoestima con el rendimiento académico en estudiantes de psicología. ingresantes el año 1999?

\section{Antecedentes}

Pintrich ( 1989) encontró que las estrategias de aprendizaje de esfuerzo, metacognición y orden presentan una alta relación el rendimiento y en lo que respecta a motivación la mayor correlación se presenta en la escala de autoeticacia.

Pintrich y García (1991) encontraron que existen bajas correlaciones entre el factor ayuda 
y el rendimiento académico, y que las escalas de elaboración, auto interrogación y metacognición están altamente relacionadas con la motivación intrínseca.

Roces, Tourón y González (1995) investigaron las estrategias de aprendizaje. motivación y rendimiento en estudiantes universitarios. encontrando diferencias entre la motivación y las estrategias utilizadas por los alumnos de mayor y menor rendimiento, siendo más marcadas las diferencias en el uso de estrategias que en las de motivación.

Álvarez y Tena (1996) investigaron la metacognición y su relación con el rendimiento académico en química general en estudiantes universitarios, los resultados indican la existencia de correlación positiva entre las variables estudiadas.

Roces, González y Tourón ( 1997) en un estudio real izado con estudiantes universitarios encontraron que las expectativas de aprendizaje y de rendimiento son dos realidades diferentes. los alumnos se creen capaces de aprender los contenidos de las distintas asignaturas y sin embargo no se creen capaces de rendir adecuadamente.

Manassero y Vázquez (1997) en sus estudios sobre motivación de logro concluyen que las atribuciones causales de los éxitos y fracasos escolares representan el eje central de la motivación de logro.

Nuñez, y cols. (1998) Investigaron sobre estrategias de aprendizaje, autoconcepto y rendimiento académico.

Los resultados expresan la existencia de diferencias significativas en la selección y utilización de estrategias de aprendizaje entre alumnos con un autoconcepto positivo y negativo y, en segundo lugar, que la relación entre el autoconcepto y las estrategias de aprendizaje es de carácter recíproco Amezcua y Fernández (1998) investigaron la influencia del autoconcepto en el rendimiento académico Los resultados obtenidos indican que la variable Autoconcepto Académico es la mejor predictora de todas las variables dependientes consideradas. seguida de la variable Autoconcepto Académico percibido de los profesores .

Bañuelos (1998) estudió las auto percepciones de habilidad y esfuerzo académico, en situaciones hipotéticas de éxito y fracaso escolar. Los resultados muestran diferencias significativas de las autopercepciones de habilidad / esfuerzo de acuerdo con la situación de éxito o fracaso. Estos hallazgos indican que un fracaso académico es atribuido a falta de esfuerzo, mientras que en una situación de éxito se confiere igual peso a la habilidad y al esfuerzo.

Roces, el. al. (1999) concluyeron que las correlaciones entre estrategias de aprendizaje con el rendimiento son más elevadas que las correlaciones con la motivación. En cuanto a las estrategias de aprendizaje sólo las estrategias de organización no se relacionan significativamente con el rendimiento y la motivación extrínseca no se relaciona con la puesta en práctica de estrategias de aprendizaje.

Aliaga et al. (2001) estudiaron las variables psicológicas relacionadas con el rendimiento académico en matemática y estadística en alumnos de primer y segundo año de la facultad de Psicología de la UNMSM, encontrando que la variable motivacional valor de la tarea, 
tiene un papel pequeño pero apreciable en el rendimiento de ambas asignaturas, y que las variables de estrategias de aprendizaje: metacognición y auto interrogatorio tienen un papel importante en el rendimiento de matemática y la variable búsqueda de ayuda con el rendimiento en estadística

\section{Objetivos}

* Conocer los componentes motivacíonales y de estrategias utilizadas por los alumnos de psicología en los que obtienen una mayor y menor puntuación

* Establecer las diferencias entre motivación, estrategias de aprendizaje y autoestima de alumnos de psicología de diferente nivel de rendimiento.

* Identiticar los factores relacionados (motivación, estrategias de aprendizaje y autoestima) con el rendimiento académico de estudiantes de psicología

\section{Variables}

Las variables a relacionar son:

motivación, estrategias de aprendizaje, autoestima y rendimiento académico.

\section{MÉTODO}

\section{Muestra}

La muestra estuvo constituida por 91 estudiantes ingresantes el año 1999, 58 estudiantes de sexo femenino. 33 de sexo masculino con una edad promedio de 21 años.

\section{Instrumentos de recolección de datos}

Para la evaluación de las estrategias de aprendizaje y motivación se utilizó el "cuestionario de estrategias de aprendizaje y motivación (CEAM) traducido y adaptado al castellano del MSLQ (Pintrich y cols. 1991) por Cristina Roces, Tourón y González) consta de 81 ítems: 31 de motivación y 50 de estrategias de aprendizaje.

Para la presente investigación se realizó el análisis de confiabilidad del cuestionario de aprendizaje y motivación. encontrándose que las escalas de Motivación presentan índices alfa de cronbach que van de 0.67 a 0.78 que se consideran muy buenos. Por otro lado, las escalas de estrategas de aprendizaje presentan índices que van de $\mathrm{O} .48$ a 0.82. Los índices más bajos corresponden a las escalas de tiempo y ayuda.

El cuestionario consta de seis escalas de motivación y siete de estrategias de aprendizaje: las escalas de motivación son las siguientes:

1. Orientación a metas intrínsecas: se refiere a la percepción de las razones por las que un estudiante se implica en una tarea de aprendizaje. Tener una motivación intrínseca en una determinada tarea académica, quiere decir que la participación de estudiante en ella es un medio más que un fin en sí mismo. items: 1, 16,22 Y 24.

2. Orientación a metas extrínsecas: grado que el estudiante percibe que participa en una tarea por razones como las notas, recompensas, la opinión de los otros o el superar al resto de sus compañeros, es decir es una orientación a los resultados, más que a la tarea. items: $7,11,13$ y 30 .

3. Valor de la tarea: opinión del alumno sobre la importancia, interés y utilidad de las asignaturas, si se consideran importantes para la propia formación, útiles para entender otras asignaturas e interesantes para ambos fines. items 4,10,17,23,26 Y 27. 
4. Creencias de control y auto eficacia para el aprendizaje: refleja hasta que punto el estudiante cree que su dominio de las asignaturas depende de su propio esfuerzo y de su modo de estudiar y hasta qué punto se considera capaz de aprender lo requerido en las distintas materias. items: 2,6,9,1215,18,25 Y 29.

5. Auto eficacia para el rendimiento: referida a las creencias de los estudiantes sobre su propia capacidad para alcanzar un buen rendimiento académico. Items: 5,20,21,3 1 .

6. Ansiedad: se refiere a los pensamientos negativos de los alumnos durante los exámenes, que interfieren en los resultados y a las reacciones fisiológicas que se ponen de manifiesto durante la realización de la prueba. Items3, 8, 14, 19, Y 28.

\section{Escalas de estrategias de aprendizaje:}

1. Elaboración: se refiere a si el alumno

aplica conocimientos anteriores a situaciones nuevas para resolver problemas, tomar decisiones o hacer evaluaciones críticas y si se establece conexiones de la información nueva con la que ya tenía para recordarla mejor. items 47,51 ,53,62,64,66,69,71 y 81,

2. Aprovechamiento del tiempo y concentración: refleja el uso que el alumno hace de su tiempo de estudio y a la capacidad que tiene para centrarse en aquello que está real izando en cada momento. items: $33,37,43,52,57,60,74,77$ y 80 .

3. Organización: se refiere al empleo de estrategias por parte del alumno para afrontar el estudio de la materia y seleccionar la información relevante como esquemas, resúmenes, subrayado etc. Items: 32,42,49, 54,59, 63,67 y 72.

4. Ayuda: referido a la ayuda que se pide a otros alumnos y al profesor durante la realización de las tareas académicas. items: $34,40,45,50,58,68$ y 75.

5. Constancia: refleja la diligencia y el esfuerzo para llevar al día las actividades y trabajos de las diferentes asignaturas y alcanzar las metas establecidas. items: 48,70,73

6. Metacognición: se refiere a la autorregulación metacognitiva, en concreto al establecimiento de metas y a la regulación del estudio y de la propia comprensión. Items:41 ,44,56,61,76,78 y 79 .

7. Autointerrogación: se refiere a las preguntas que el alumno se hace a si mismo durante el estudio, con el objeto de centrarse, examinar su nivel de comprensión y cuestionar la veracidad de lo estudiado. Items:36,38 y 55.

Escala de autoestima de M. Rosenberg Está constituida por 10 ítems positivos y negativos que se alternan, la con fiabilidad fue estudiada a través del coeficiente de reproductibilidad de la escala tipo Guttman, la cual ascendió a 0.92 y el coeficiente de escalabilidad de 0.73 . Estudios realizados por Silber y Tippett (Rosemberg, 1987) demostraron que la escala tenía una con fiabilidad test retest de 0.85 con un intervalo de dos semana entre cada aplicación.

\section{RESULTADOS}

\section{Motivación: valores medios de las escalas de motivación y estrategias de aprendizaje}

Los puntajes medios de las escalas de motivación, de mayor a menor son: las creencias de control, el valor de la tarea, la autoeficacia para el rendimiento, orientación a metas intrínsecas, orientación a metas extrínsecas y en último lugar la ansiedad. Las creencias de control y valor dc la. tarea ocupan el primer y segundo lugar, lo que significa la valoración del esfuerzo personal para lograr el dominio de las asignaturas los aprendizajes esperados, aSImismo el fracaso es asociado a la falta de esfuerzo, aspecto muy positivo por cuanto el esfuerzo es una variable interna que puede ser controlada por el sujeto y en cuanto al valor de la tarea que significa la opinión del alumno sobre la importancia, interés y utilidad de las asignaturas. 
La media más baja (3.27) es en la escala de ansiedad, lo que resultaría positivo dado que la ausencia de pensamientos ansiosos y reacciones fisiológicas asociadas durante el examen permite al alumno una mayor concentración y mayor rendimiento en el mismo.

Con relación a las estrategias de aprendizaje, los puntajes medios de mayor a menor son: constancia, metacognición, organización, elaboración y auto interrogación y en últimos lugares ayuda, tiempo y concentración.

Como se muestra también en los gráficos 1 y 2 los estudiantes de psicología admiten mayor frecuencia de motivación relacionada con Creencias de control y valor de la tarea pero menor frecuencia en expresiones relacionadas con Ansiedad; mayor frecuencia de estrategias de constancia y metacognición y menor frecuencia de tiempo y ayuda.

\section{Tabla 1:}

Valores medios de las escalas motivacionales. de las estrategias de aprendizaje y de la autoestima

\section{Valor Medio}

Motivación

Auto eficacia

Ansiedad

Valor de la

tarea

Metas

extrínsecas

Metas

intrínsecas

Creencias

Control
5.4

Estrategias de Aprendizaje

Elaboración

Tiempo y

concentración

Ayuda

Organización

Constancia

Metacognición

Auto interrogación 


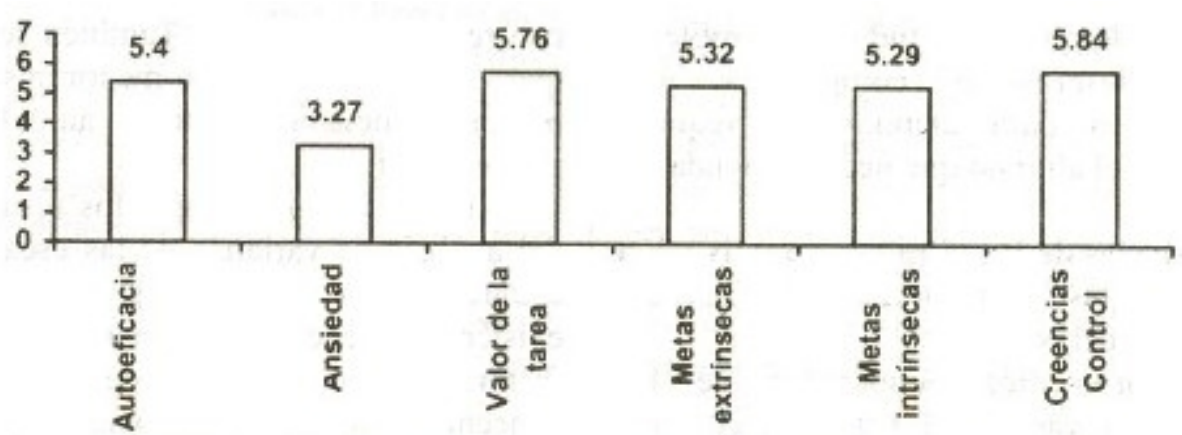

Gráfico 1. Puntajes medios en las subescalas de Motivación para los estudiantes de psicologia.



Gráfico 2. Puntajes medios en las escalas de Estrategias de Aprendizaje para los estudiantes de psicologia.

\section{Aspectos motivacionales y de estrategias de aprendizaje con puntuaciones más y menos valorados.}

Los estudiantes de psicología valoran más expresiones de creencias de control, metas extrínsecas y valor de la tarea y valoran menos expresiones de Ansiedad, lo cual puede ser explicable por la carrera que estudian. En cuanto a las creencias de control y autoeficacia para el aprendizaje señala un aspecto muy importante que es el reconocimiento de su capacidad para aprender SI realiza los esfuerzos necesarios.

En cuanto a las estrategias de aprendizaje, los estudiantes de psicología valoran más expresiones de Constancia, Metacognición y Organización y valoran menos expresiones de Tiempo y Ayuda. El item de constancia puede resultar engañoso si tenemos en cuenta la obligatoriedad de la asistencia como requisito para aprobar los cursos. Si comparamos los ítems más y menos valorados resultan contradictorios los resultados en estrategias de aprendizaje por cuanto valoran aspectos referidos al esfuerzo y la autorregulación; pero asimismo no utilizan el tiempo en forma adecuada ni solicitan la ayuda correspondiente cuando la requieren; obviamente éste último punto puede estar relacionado con la actitud desfavorable de los profesores o compañeros más adelantados para atender los requerimientos del alumno que necesita ayuda.

3. Niveles de Rendimiento, motivación y estrategias de aprendizaje: análisis de las diferencias

Los promedios ponderados de los estudiantes van de 11.3 a 16.6, con una media de 14.4 y 
una desviación están dar de 1.05. El percentil 33 es 13.9 y el percentil 66 es 14.8. Siguiendo a Roces, Tourón y González (1995) se formo tres niveles de rendimiento considerando dichos percentiles como puntos de corte. En las tablas siguientes se presentan análisis de varianza para probar si los grupos de rendimiento que pueden formarse difieren en las escalas de motivación y estrategias de aprendizaje.

En la Tabla 4 se presentan los resultados correspondientes al análisis de varianza de las escalas motivacionales. Solamente existen diferencias significativas en un factor: Orientación a metas extrínsecas. Las diferencias significativas se dan entre los grupos 1 y 3. Se trata, como se puede ver en la figura 3, de un perfil lineal pero descendente. es decir los alumnos con alto rendimiento valoran menos las metas extrínsecas que los alumnos de bajo rendimiento. Esto se puede observar mejor en la tabla 5 analizando las medias correspondientes a los diferentes grupos.

Aunque las diferencias entre los grupos en el resto de las escalas no son significativas. podemos observar en la gráfica 3 que, en las escalas de autoeficacia para el rendimiento, valor de la tarea y orientación a metas intrínsecas hay un valor medio más alto en el grupo con rendimiento medio. También se tiene que a mayor rendimiento menor ansiedad, menor creencias de control y autoeficacia para el aprendizaje.

En la tabla 6 se recogen los resultados del análisis de varianza de las escalas de estrategias de aprendizaje. Solamente existen diferencias significativas en un factor: aprovechamiento del tiempo y concentración. Las diferencias significativas se dan entre los grupos 1 y 3 . Se trata, como se puede ver en la figura 4, de un perfil lineal pero descendente, es decir los alumnos con alto rendimiento valoran menos el aprovechamiento del tiempo y la concentración que los alumnos de bajo rendimiento. Esto se puede observar mejor en la tabla 7 analizando las medias correspondientes a los diferentes grupos.

Aunque las diferencias entre los grupos en el resto de las escalas no son significativas. podemos observar en la gráfica 4 que en las escalas de Elaboración, Ayuda y auto interrogación hay un valor medio más alto en el grupo con rendimiento medio. También se tiene que a mayor rendimiento menor aprovechamiento del tiempo y control, pero también a mayor rendimiento mayor Organización, constancia y metacognición.

No se ha encontrado diferencias significativas en la autoestima con relación al rendimiento (tabla8). Sin embargo se observa que a mayor $r$ rendimiento mayor Autoestima.

\section{Relación del Rendimiento con Motivación, Estrategias de Aprendizaje y Autoestima} La tabla 9 muestra que las correlaciones significativas e inversas con el rendimiento académico son las motivaciones referidas a orientación a metas extrínsecas y ansiedad y la estrategia de aprendizaje que se refiere a aprovechamiento del tiempo y concentración. Por el contrario la constancia se relaciona positivamente con el rendimiento. 
Tabla 2: Items de motivación más y menos valorados

\begin{tabular}{cccl}
\hline Escalas & $\mathbf{N}^{\mathbf{0}}$ & Media & \multicolumn{1}{c}{ Items más valorados (mayor y menor) } \\
\hline Creencias control & 12 & 6.37 & Estoy seguro de aprender los conceptos básicos \\
Creencias control & 18 & 6.34 & Si me esfuerzo entenderé los contenidos \\
Creencias control & 2 & 6.33 & Si estudio del modo adecuado, aprenderé los contenidos \\
Metas extrínsecas & 13 & 6.20 & Si puedo, quiero sacar las mejores notas que la mayoría \\
Valor Tarea & 23 & 5.98 & Creo que es útil para mi aprenderme las asignaturas \\
Ansiedad & 03 & 2.50 & Cuando hago un examen, pienso que esta saliendo peor \\
Ansiedad & 28 & 3.30 & Cuando hago un examen, mi pulso se acelera \\
Ansiedad & 19 & 3.36 & Me siento nervioso y turbado cuando hago exámenes \\
Ansiedad & 08 & 3.53 & Mientras hago un examen, pienso continuamente \\
Ansiedad & 14 & 3.68 & Mientras hago un examen, pienso en las consecuencias
\end{tabular}

Tabla 3: Items de estrategias más y menos valorados

\begin{tabular}{cccl}
\hline Escalas & $\mathbf{N}^{\mathbf{0}}$ & Media & \multicolumn{1}{c}{ Items más valorados (mayor y menor) } \\
\hline Constancia & 73 & 6.187 & Asisto a clase con regularidad \\
Metacognición & 41 & 5.780 & Si leo vuelvo atrás para aclarar dudas \\
Metacognición & 79 & 5.725 & Si me pierdo tomando apuntes procuro completarlos \\
Organización & 32 & 5.593 & Subrayo para organizar mejor mis ideas \\
Metacognición & 76 & 5.528 & Cuando estudio, trato de determinar que conceptos no entiendo bien \\
Constancia & 70 & 5.517 & Procuro estar al día en el estudio y los trabajos \\
Organización & 42 & 5.473 & Cuando estudio hago una lectura rapida e intento encontrar las ideas principales \\
Tiempo & 60 & 2.978 & Cuando la materia es difícil abandono o estudio las partes mas fáciles \\
Ayuda & 34 & 3.439 & Expongo ante compañeros la materia para comprobar lo que se \\
Tiempo & 57 & 3.484 & Frecuentemente leo pero sin enterarme de lo que leo \\
Tiempo & 52 & 3.615 & Encuentro difícil ceñirme a un plan de estudio \\
Ayuda & 58 & 3.659 & Hago preguntas a los profesores para aclarar \\
Tiempo & 37 & 3.879 & Muchas veces me aburro tanto cuando estudio \\
Tiempo & 80 & 4.099 & Me cuesta encontrar tiempo para repasar
\end{tabular}

\section{Tabla 4. Análisis de varianza, comparaciones multiples, análisis de tendencias de la relación de los distintos factores motivacionales con relacion al rendimiento académico}

\begin{tabular}{|c|c|c|c|}
\hline \multirow[t]{2}{*}{ Factores } & \multicolumn{2}{|c|}{$\begin{array}{c}\text { Análisis de } \\
\text { Varianza }\end{array}$} & \multirow[t]{2}{*}{$\begin{array}{c}\text { Comparación de } \\
\text { múltiples significativas }\end{array}$} \\
\hline & $\mathrm{F}$ & $\mathrm{P}$ & \\
\hline Autosuficiencia para el rendimiento & 1.03 & 0.360 & \\
\hline Ansiedad & 2.73 & 0.070 & \\
\hline Valor de la tarea & 0.03 & 0.970 & $1-3$ \\
\hline Orientación a metas Extrínsecas & 3.44 & 0.040 & \\
\hline Orientación a metas Intrínsecas & 0.97 & 0.380 & \\
\hline Creencias de control y autosuficiencia para el aprendizaje & 0.47 & 0.630 & \\
\hline
\end{tabular}


Tabla 5. Valores medios de las escalas motivacionales del cuestionario en relacion con los tres grupos de rendimiento formados

\begin{tabular}{lcccccc}
\hline \multicolumn{1}{c}{ Factores } & \multicolumn{2}{c}{ Rendimiento } & \multicolumn{2}{c}{ Rendimiento } & \multicolumn{2}{c}{ Rendimiento } \\
& \multicolumn{2}{c}{ Bajo } & & Bajo & \multicolumn{2}{c}{ Bajo } \\
\hline & Media & D.E. & Media & D.E. & Media & D.E. \\
\hline Autosuficiencia para el rendimiento & 5.26 & 0.97 & 5.59 & 0.87 & 5.36 & 0.93 \\
Ansiedad & 3.66 & 1.27 & 3.27 & 1.30 & 2.89 & 1.28 \\
Valor de la tarea & 5.74 & 0.74 & 5.79 & 0.92 & 5.76 & 0.74 \\
Orientación a metas Extrínsecas & 5.71 & 1.02 & 5.30 & 1.01 & 4.96 & 1.29 \\
Orientación a metas Intrínsecas & 5.33 & 0.95 & 5.46 & 1.02 & 5.09 & 1.15 \\
Creencias de control y autosuficiencia para el aprendizaje & 5.93 & 0.63 & 5.85 & 0.67 & 5.75 & 0.75 \\
\hline
\end{tabular}

Gráfico 3: Valores medios de las escalas de motivación

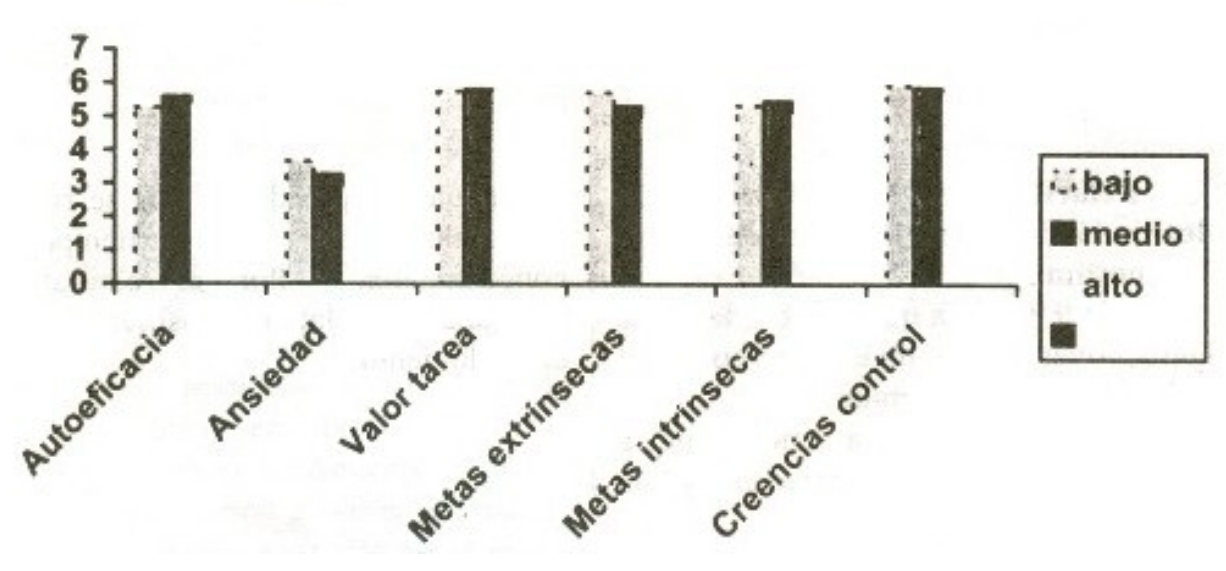

Tabla 6. Análisis de varianza, comparaciones múltiples, análisis de tendencias de la relación de los distintas escalas de estrategias de aprendizaje en relación al rendimiento educativo.

\begin{tabular}{lcccc}
\hline \multicolumn{1}{c}{ Factores } & \multicolumn{2}{c}{$\begin{array}{c}\text { Análisis de } \\
\text { varianza }\end{array}$} & \multirow{2}{c}{$\begin{array}{c}\text { Comparaciones múltiples } \\
\text { Significativas }\end{array}$} \\
\cline { 1 - 3 } Elaboración & F & P & \\
Aprovechamiento del tiempo y concentración & 0.25 & 0.78 & \\
Ayuda & 4.75 & $0.01^{*}$ & \\
Organización & 1.23 & 0.30 & \\
Constancia & 0.33 & 0.72 & \\
Metacognición & 2.90 & 0.06 & \\
Autointerrogación & 1.95 & 0.15 & \\
\hline
\end{tabular}


Tabla 7. Valores medio de las escalas de estrategias de aprendizaje del cuestionario en relación con los tres grupos de rendimiento formados.

\begin{tabular}{lcccccc}
\hline \multirow{2}{*}{ Escala de Estrategias de Aprendizaje } & \multicolumn{2}{c}{$\begin{array}{c}\text { Rendimiento } \\
\text { Bajo }\end{array}$} & \multicolumn{2}{c}{$\begin{array}{c}\text { Rendimiento } \\
\text { Bajo }\end{array}$} & \multicolumn{2}{c}{$\begin{array}{c}\text { Rendimiento } \\
\text { Bajo }\end{array}$} \\
\hline & Media & D.E. & Media & D.E. & Media & D.E. \\
\hline Elaboración * & 4.90 & 1.02 & 5.06 & 0.86 & 4.94 & 0.81 \\
Aprovechamiento del tiempo y concentración & 4.38 & 0.62 & 4.18 & 0.75 & 3.85 & 0.63 \\
Ayuda * & 4.18 & 0.83 & 4.49 & 0.68 & 4.33 & 0.81 \\
Organización & 4.90 & 0.97 & 4.97 & 0.87 & 5.09 & 0.93 \\
Constancia & 5.20 & 1.26 & 5.76 & 1.01 & 5.74 & 0.78 \\
Metacognición & 5.04 & 0.74 & 5.38 & 0.72 & 5.35 & 0.76 \\
Autointerrogación * & 4.67 & 1.51 & 4.98 & 1.10 & 4.58 & 1.04 \\
\hline
\end{tabular}

3. Niveles de rendimiento y autoestima: análisis de las diferencias

Tabla 8. Análisis de varianza de Autoestima en relación al rendimiento académico.

\begin{tabular}{lcccccc}
\hline Escala & \multicolumn{2}{c}{ Rendimiento Bajo } & \multicolumn{2}{c}{ Rendimiento medio } & \multicolumn{2}{c}{ Rendimiento alto } \\
& Media & D.E. & Media & D.E. & Media & D.E. \\
& & & & & & \\
\hline Autoestima & 13.70 & 3.77 & 13.71 & 5.07 & & \\
Prueba F & 1.273 & & & & & \\
$\mathrm{P}$ & 0.285 & & & & & \\
\hline
\end{tabular}

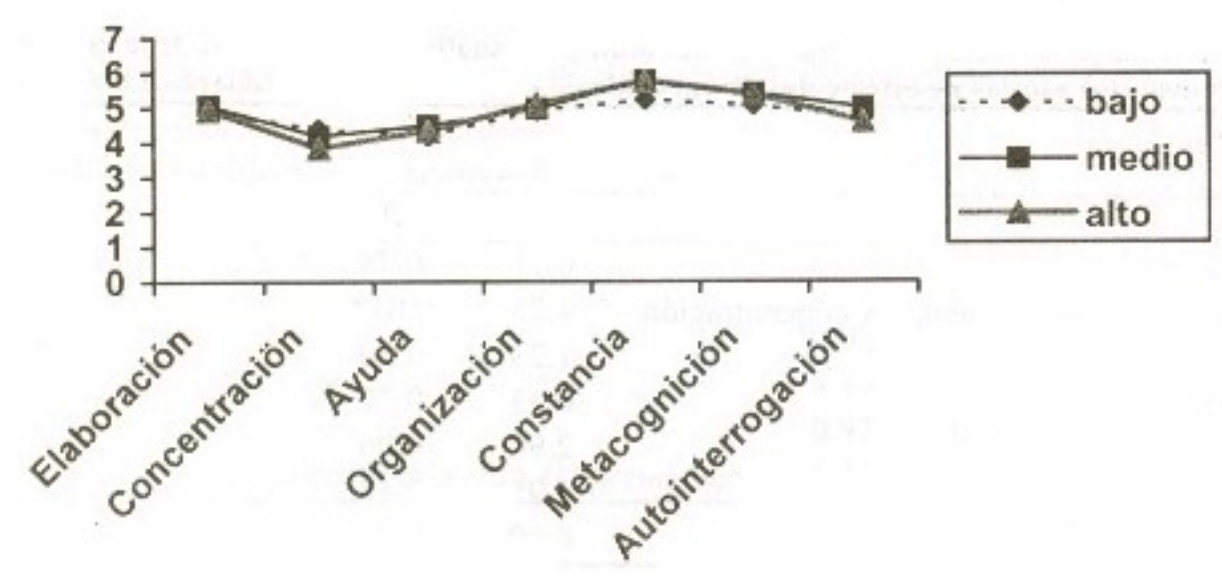




\begin{tabular}{lll}
\hline Escalas & $\mathrm{r}$ & $\mathrm{P}$ \\
\hline \multicolumn{1}{c}{ Motivación } & & \\
Orientación a metas intrínsecas & -0.09 & 0.390 \\
Orientación a metas extrínsecas & -0.27 & 0.010 \\
Valor de la tarea & 0.01 & 0.940 \\
Creencias de control y autoeficacia para el aprendizaje & -0.10 & 0.340 \\
Autoeficacia para el rendimiento & 0.04 & 0.680 \\
Ansiedad $\quad$ Estrategias de Aprendizaje & -0.24 & 0.020 \\
& & \\
Elaboración & 0.02 & 0.870 \\
Aprovechamiento del tiempo y concentración & -0.31 & 0.000 \\
Organización & 0.09 & 0.420 \\
Ayuda & 0.08 & 0.450 \\
Constancia & 0.21 & 0.040 \\
Meta cognición & 0.17 & 0.110 \\
Auto interrogación & -0.03 & 0.780 \\
Autoestima & -0.15 & 0.170 \\
\hline
\end{tabular}

Tabla 9. Correlación de Pearson de las escalas de Motivación, Estrategias de Aprendizaje, y Autoestima con el rendimiento académico.

\section{DISCUSIÓN}

En relación a la motivación, los puntajes medios más altos corresponden a las creencias de control y autoeficacia para el aprendizaje, valor de la tarea y autoeficacia para el rendimiento, aspectos que revelan la confianza del sujeto en sus posibilidades de aprender y rendir adecuadamente, así como la importancia que le conceden a las asignaturas para su propia formación y el valor medio más bajo. corresponde a la ansiedad lo que Implica un control sobre la misma lo cual es un aspecto altamente positivo. En la misma dirección encontramos los resultados en los ítems más y menos valorados.

En cuanto a las estrategias de aprendizaje, las más utilizadas son las de constancia y metacognición que refleja la diligencia y esfuerzo para cumplir con las actividades y trabajos académicos para así alcanzar las metas establecidas, asimismo la metacognición implica la autorregulación del estudio y la actividad cognitiva. Las estrategias de tiempo y ayuda alcanzan la media más baja, lo que Implica por un lado las dificultades del alumno para organizar su tiempo y aprovechado de manera eficaz y por otro lado que la solicitud de apoyo al profesor o compañeros de estudio es la menos utilizada, que puede ser debido a patrones de interacción poco comunicativos entre compañeros y profesor-alumno así como a la estructura de las clases que no permite la participación activa del alumno o al $\sim \mathrm{sca} \sim \mathrm{o}$ uso de estrategias didácticas que Impliquen el aprendizaje cooperativo. En los ítems más y menos valorados encontramos la misma tendencia.

Si analizamos los puntajes medios de motivación y estrategias de aprendizaje, resulta contradictorio encontrar alumnos que se consideran capaces de aprender y rendir, que utilizan estrategias meta cognitivas; pero que a su vez no le dedican el tiempo necesario a los aprendizajes o tareas que se le plantean y cuando requieren ayuda no la solicitan.

En el análisis de las diferencias de los grupos de distinto nivel de rendimiento respecto a 
la motivación se encontraron diferencias respecto a la escala de orientación a metas extrínsecas en el grupo de alto y bajo rendimiento asimismo respecto a las estrategias de aprendizaje ambos grupos extremos difieren en la estrategia: aprovechamiento del tiempo y concentración, el grupo de rendimiento si bien no se diferencia significativamente de los otros grupos es más homogéneo en sus valores medios tanto en las escalas de motivación como de estrategias de aprendizaje en auto eficacia para el rendimiento, valor de la tarea y orientación a metas intrínsecas y las escalas de elaboración, ayuda y auto interrogación.

Respecto a las relaciones entre motivación, estrategias de aprendizaje y autoestima con el rendimiento académico los resultados muestran escasas relaciones; de las variables motivacionales ninguna de ellas correlaciona positivamente con el rendimiento más bien se encuentra una relación inversa entre rendimiento y dos variables motivacionales: orientación a metas extrínsecas y ansiedad; es decir que a mayor rendimiento menor orientación a metas extrínsecas y ansiedad, desde un punto de vista teórico se asume como deseable para un mejor aprendizaje la orientación hacia metas intrínsecas que implica un compromiso interno vinculado a la curiosidad, el reto, el afán de conocer, contrariamente a ello la orientación a metas extrínsecas se vincula con aspectos más instrumental es como obtener buenas notas, recompensas etc.; por otro lado el mejor control de la ansiedad establece menor interferencia durante los exámenes, en éste caso los alumnos de menor rendimiento tienen mayor ansiedad, es decir tienen menor capacidad de control de la misma, resultados similares fueron hallados por Pintrich y DeGroot (1990b).

La relación entre autoestima y rendimiento académico no es significativa aún cuando existe una tendencia que expresa que a mayor autoestima mayor rendimiento; probablemente la utilización de otros instrumentos de medida de la autoestima, más orientados a la dimensión académica, pudieran arrojar - otros resultados, aún cuando en la investigación de Aliaga et.al (2001) tampoco se encontraron relaciones entre el auto concepto académico y el rendimiento.

La no existencia de correlaciones entre las variable motivacional: auto eficacia con el rendimiento académico difiere de otros trabajos realizados como los de Roces ( 1997) Pintrich et al. 1991, en el que encontraron las correlaciones más elevadas de ésta variable con el rendimiento.

Respecto a las estrategias de aprendizaje la correlación positiva entre la variable constancia y rendimiento académico es similar a los resultados de Roces ( ) y Tourón (1989) lo que implica el esfuerzo permanente del sujeto en relación a las metas que se ha propuesto: la relación inversa de la estrategia: aprovechamiento del tiempo y concentración con el rendimiento académico resulta sorprendente; pero explicable, pudiera darse el caso que los alumnos de menor rendimiento empleen más tiempo y requieran mayor concentración que los de alto rendimiento para obtener resultados esperados, sobre todo si se tiene en cuenta que la variabilidad de los promedios de nota de los alumnos de la muestra es pequeña, sobre todo si lo comparamos con otras facultades de la universidad.

Por otra parte, en nuestra universidad el promedio ponderado del alumno es resultado del total de cursos matriculados, donde se incluyen asignaturas que el alumno no cursó por motivos diferentes: horarios, trabajo, etc., seria recomendable para futuras investigaciones corregir estos sesgos que pueden afectar la ubicación del alumno en niveles de rendimiento que no le corresponden. 
Finalmente es importante pensar en la variable profesor en su relación con el rendimiento académico del alumno, considerando diferentes aspectos, tales como el uso de estrategias didácticas orientadas a la reflexión y problematización como factor importante de la motivación del alumno, congruencia entre lo que enseña y lo que evalúa, el estilo comunicacional e interactivo que establece con sus alumnos entre tantos aspectos a considerar.

\section{CONCLUSIONES}

1. Los puntajes medios más altos en motivación corresponden a creencias de control, valor de la tarea y autoeficacia para el rendimiento y el más bajo corresponde a ansiedad.

2. Los puntajes medios más altos en estrategias de aprendizaje corresponden a constancia y meta-cognición y los más bajos corresponden a tiempo y ayuda.

3. Existen diferencias significa-tivas en los grupos de alto y bajo rendimiento, respecto a la orientación a metas extrínsecas y aprovechamiento del tiempo y concentración.

4. Existe correlación significativa inversa entre la orientación a metas extrínsecas y ansiedad con el rendimiento académico.

5. Existe relación positiva entre la estrategia: constancia y el rendimiento académico.

6. No existe relación significativa entre autoestima y rendimiento académico.

\section{REFERENCIAS}

Alonso, J. (1991) Motivación y aprendizaje en el aula. Cómo enseñar a pensar. Madrid: Santillana.

Apodaca, P. y Lobato, C. (Eds.) (1997) Calidad en la universidad: orientación y evaluación. Laertes: Barcelona

García Llamas, J. (1986) El estudio empírico del rendimiento académico en la enseñanza a distancia. Madrid: ICE.

Gonzales, D. (1995) Teoría de la motivación y práctica profesional. La Habana:Pueblo y educación

Granell, E.; Viyas, E, Feldman, L. (1993) Rechazo escolar. México: Trillas Huertas, J. (1997) Motivación. Querer aprender. Buenos Aires: Aiqué

Latiesa, M. (1992) La deserción universitaria. Madrid: CIS.

Oyola, C.; Barila,M.; Figueroa,E. (1997) Fracaso escolar. El éxito prohibido. Buenos Aires: Aiqué

Manassero,M. Y Vázquez, A. (1997) Análisis empírico de dos escalas de motivación escolar. Revista electrónica de motivación y emoción. Volumen $3 \mathrm{~N}^{\circ}$ 5-6

Manassero, M. A. ; Vázquez Alonso, A. (1995a). La atribución causal y la predicción del logro escolar: Patrones causales, dimensionales y emocionales. Estudios de Psicología, 54, $3-22$.

Pintrich,P. Y Garcia, T. (1991) Individual differences in student motivational orientation, self regulatión in the college clasroom. En Pintrich y Maher (Eds) advances in motivatión and achievement. Vo1.7. Greenwich, CT: Jai Press.

Manassero, M. A. ; Vázquez Alonso, A. (1995b).La atribución causal como determinante de las expectativas. Psicothema, 7, 361-376.

Marchesi, A. y Martín, E.( 1999) Calidad de la enseñanza en tiempos de cambio. Madrid: Alianza editorial.

Monereo, C. (Coordinador) (1997) Estrategias de enseñanza y aprendizaje. Barcelona: Grao 
Navas, I.; sampascual, g. y castejón, j.1. (1991): las expectativas de profesores y alumnos como predictores del rendimiento académico. Revista de psicología general y aplicada. Vol. 44 (2), pp 231-239.

Navas, I.; sampascual, g. y castejón, j.1. (1992) Atribuciones y expectativas de alumnos y profesores. Influencias en el rendimiento escolar. Revista de Psicología General y Aplicada. Vol. 45 (1) pp 55-56.

Nisbet, J. Y Shucksmith, J. (1986) Estrategias de aprendizaje. Madrid: Santillana

Núñez; J.C., González-Pienda, J.A., García, M., González-Pumariega, S., Roces, e., Álvarez, L. y González, M.e. (1998). Estrategias de aprendizaje, autoconcepto y rendimiento académico. Psicothema, 10 ( 1), 97-109.

Page, A. (1990) Hacia un modelo causal del rendimiento académico. Madrid: CIDE.

Roces, e. Et al. Relaciones entre motivación, estrategias de aprendizaje y rendimiento académico en estudiantes universitarios. En Mente y conducta en situación educativa. Revista electrónica del Departamento de psicología de la U. de Valladolid. Volumen 1 Número 1: 4150

Roces, e. (1996) Estrategias de aprendizaje y motivación en la Universidad. Tesis doctoral inédita Universidad de Navarra

Roces, C., González, M.e. y Tourón, J. (1997). Expectativas de Aprendizaje y de Rendimiento de los Alumnos Universitarios. Revista de Psicología de la Educación, 22, 99123.

Rodríguez Espinar, S. (1982). Factores de rendimiento Escolar. Barcelona: OikosTau.

Rosenberg,M. (1973) La auto imagen del adolescente y la sociedad. Buenos Aires: Paidos

Tourón, J. (1989). Métodos de Estudio en la Universidad. Pamplona: Eunsa. 